\title{
An Overview of the Multi-Band and the Generalized BCS Equations-Based Approaches to Deal with Hetero-Structured Superconductors
}

\author{
G. P. Malik ${ }^{*}$ \\ Theory Group, School of Environmental Sciences, Jawaharlal Nehru University, New Delhi, India \\ Email:gulshanpmalik@yahoo.com,malik@mail.jnu.ac.in
}

How to cite this paper: Malik, G.P. (2018) An Overview of the Multi-Band and the Generalized BCS Equations-Based Approaches to Deal with Hetero-Structured Superconductors. Open Journal of Microphysics, 8, 7-13.

https://doi.org/10.4236/ojm.2018.82002

Received: March 10, 2018

Accepted: May 8, 2018

Published: May 11, 2018

Copyright $\odot 2018$ by author and Scientific Research Publishing Inc. This work is licensed under the Creative Commons Attribution International License (CC BY 4.0).

http://creativecommons.org/licenses/by/4.0/

\section{(c) (i) Open Access}

\begin{abstract}
We trace the conceptual basis of the Multi-Band Approach (MBA) and recall the reasons for its wide following for composite superconductors (SCs). Attention is then drawn to a feature that MBA ignores: the possibility that electrons in such an SC may also be bound via simultaneous exchanges of quanta with more than one ion-species-a lacuna which is addressed by the Generalized BCS Equations (GBCSEs). Based on several papers, we give a concise account of how this approach: 1) despite employing a single band, meets the criteria satisfied by MBA because a) GBCSEs are derived from a temperature-incorporated Bethe-Salpeter Equation the kernel of which is taken to be a "superpropagator" for a composite SC-each ion-species of which is distinguished by its own Debye temperature and interaction parameter and b) the band overlapping the Fermi surface is allowed to be of variable width. GBCSEs so-obtained reduce to the usual equations for the $T_{c}$ and $\Delta$ of an elemental SC in the limit superpropagator $\rightarrow 1$-phonon propagator; 2) accommodates moving Cooper pairs and thereby extends the scope of the original BCS theory which restricts the Hamiltonian at the outset to terms that correspond to pairs having zero centre-of-mass momentum. One can now derive an equation for the critical current density $\left(j_{0}\right)$ of a composite SC at $T=0$ in terms of the Debye temperatures of its ions and their interaction parameters-parameters that also determine its $T_{c}$ and $\Delta s, 3$ ) transforms the problem of optimizing $j_{0}$ of a composite SC, and hence its $T_{\mathcal{c}}$ into a problem of chemical engineering, 4) provides a common canopy for most composite SCs, including those that are usually regarded as outside the purview of the BCS theory and have therefore been called "exceptional", e.g., the heavy-fermion SCs; 5) incorporates $s^{ \pm}$-wave superconductivity as an in-built feature and can therefore deal with the iron-based SCs, and 6) leads to presumably verifiable
\end{abstract}

*Present address: B 208 Sushant Lok 1, Gurgaon 122009, Haryana, India. 
predictions for the values of some relevant parameters, e.g., the effective mass of electrons, for the SCs for which it has been employed.

\section{Keywords}

Hetero-Structured Superconductors, Multi-Band and Generalized BCS Equations-Based Approaches, Unified Treatment of Cuprates and Fe-Based Superconductors, Quest for Clues about Raising $T_{c}$

\section{Introduction}

We trace in Section 2 the backdrop of Multi-Band Approach (MBA) for hetero-structured, multi-gapped superconductors (SCs) based on numerous papers, for the gist of which [1] [2] [3] suffice. Gleaned from [1], summarized then are the reasons for its wide adoption. In Section 3, based on [4] [5] [6] [7] and [8], is given an account of the Generalized-BCS Equations (GBCSEs)-based approach (CA henceforth because it complements MBA), which also has been valuably employed to deal with such SCs. The last section is devoted to a discussion of the salient distinguishing features of the two approaches and conclusion.

\section{MBA}

At the root of MBA is the work of Suhl et al. [2] who dealt with the superconductivity of transition elements for which the occupation of the $4 \mathrm{~s}$ orbitals begins prior to complete filling of the $3 \mathrm{~d}$ orbitals, leading to division of valence electrons between two bands. Pairing can therefore also be caused by cross-band scattering. Because the d-band has more vacant levels than the s-band, it makes a large contribution to the total density of states $N(0)$. Two gaps and, in general, two $T_{\mathcal{c}}$ arise in this approach because the BCS interaction parameter $\lambda \equiv$ $[N(0) V]$ is now determined not via a single interaction energy " $V$ ", but via a quadratic equation involving three such energies: $V_{s}$ and $V_{d}$ due to scattering in the two bands individually and $V_{s d}$ due to cross-band scattering. Since in this model the equation employed to determine $T_{c}$-for each value of $\lambda$-is the familiar BCS equation for elemental SCs derived for one-band, weak-coupling $(\lambda$ $<0.5)$ theory, it cannot per se explain the occurrence of high- $T_{c} s$. For this reason, the multi-band concept is supplemented by the well-known Migdal-Eliashberg-McMillan approach [3], which allows $\lambda$ to be greater than even unity because it is based on an integral equation the expansion parameter of which is not $\lambda$, but $\left(m_{e} / M\right)$, where $m_{e}$ is the mass of an electron and $M$ that of an ion. MBA has evolved around these basic ideas because anisotropic SCs necessitate that [1]: 1) the BCS assumption of $E_{F} \gg k \theta$ ( $E_{F}=$ Fermi energy, $k=$ Boltzmann constant; $\theta=$ Debye temperature) be abandoned; 2) different locations in k-space should be characterized by distinct pairing strengths and order parameters (i.e., gaps); and 3) the assumption that the Fermi surface is isotropic/spherical be dispensed with. 
Indeed, numerous SCs have been listed in [1] for which MBA has found a useful application.

\section{CA: Physical Basis [4]}

A striking feature of all SCs that have $T_{c}$ s greater than that of $\mathrm{Nb}(\approx 9 \mathrm{~K})$ is that they are multi-component materials, suggesting naturally that Cooper pairs (CPs) in them may also be bound via simultaneous exchanges of phonons with more than one species of ions. It has been shown [4] that the BCS equation for $T_{c}$ of an elemental SC can also be obtained via a Bethe-Salpeter equation (BSE) with a kernel corresponding to the one-phonon exchange mechanism (1PEM) in the ladder approximation. The first diagram in this series has one rung, the second two rungs, and so on. If the number of rungs between any two space-time points in each of these diagrams is doubled, then we have the 2-phonon exchange mechanism (2PEM) in operation. Similarly-depending on composition of the SC-CPs may also be bound via a 3-phonon exchange mechanism (3PEM). It hence follows that in a composite SC, CPs may exist with different values of the binding energy $(2|W|)$. Since the inequalities $\left|W_{3 \text { PEM }}\right|>\left|W_{2 \text { PEM }}\right|>\left|W_{1 \text { PEM }}\right|$ must hold, and since $|W|=\Delta[4]$, we are naturally led to an explanation of why multi-component SCs are characterized by multiple gaps.

\subsection{GBCSEs Incorporating Chemical Potential in the 2PEM Scenario}

Employing

$I(\boldsymbol{p})=-\left(V_{1}+V_{2}\right) /\left(2 \pi^{3}\right)$

$\left(\hbar=c=1 ; V_{i}=0\right.$, except when $\left.E_{F}-k \theta_{i} \leq p^{2} / 2 m^{*} \leq E_{F}+k \theta_{i}\right)$

as the kernel of a

BSE, the following $E_{F}$-incorporated equations have been derived for $\left|W_{20}\right|$ (to be identified with $\Delta_{2}>\Delta_{1}$ ) and $T_{\mathcal{c}}$ where $\Delta_{1}$ and $\Delta_{2}$ are any two gap-values of an SC which may also be characterized by additional $\Delta$-values [4] [5]:

$$
\begin{aligned}
& \operatorname{Re}\left\{\frac{\lambda_{1}}{2} \int_{-k \theta_{1}}^{k \theta_{1}} \mathrm{~d} \xi \frac{\sqrt{\xi+\mu}}{|\xi|+\left|W_{20}\right| / 2}+\left(\left|W_{20}\right| ; \lambda_{1} \rightarrow \lambda_{2} ; \theta_{1} \rightarrow \theta_{2}\right)\right\} \\
= & \operatorname{Re}\left\{\left[\left(\mu-k \theta_{2}\right)^{3 / 2}+\frac{3}{4} \int_{-k \theta_{2}}^{k \theta_{2}} \mathrm{~d} \xi \sqrt{\xi+\mu}\left(1-\frac{\xi}{\sqrt{\xi^{2}+W_{20}^{2}}}\right)\right]^{1 / 3}\right\} \\
& \operatorname{Re}\left\{\frac{\lambda_{1}}{2} \int_{-k \theta_{1}}^{k \theta_{1}} \mathrm{~d} \xi \frac{\sqrt{\xi+\mu} \tanh \left(\xi / 2 k T_{c}\right)}{\xi}+\left(T_{c} ; \lambda_{1} \rightarrow \lambda_{2} ; \theta_{1} \rightarrow \theta_{2}\right)\right\} \\
= & \operatorname{Re}\left\{\left[\frac{3}{4} \int_{-\mu}^{k \theta_{2}} \mathrm{~d} \xi \sqrt{\xi+\mu}\left\{1-\tanh \left(\xi / 2 k T_{c}\right)\right\}\right]^{1 / 3}\right\}
\end{aligned}
$$

where chemical potential $\mu$ has been used interchangeably with $E_{F}, \theta_{1}$ and $\theta_{2}>$ $\theta_{1}$ are the Debye temperatures of the ion-species that cause pairing and $\lambda_{1} \equiv\left[N(0) V_{1}\right]$ and $\lambda_{2} \equiv\left[N(0) V_{2}\right]$ their interaction parameters, no distinc- 
tion is made between the values of $\mu$ and the $\lambda \mathrm{s}$ at $T=0$ and $T=T_{\mathcal{c}}$ and $R e$ ensures that the integrals yield real values even when $\mu<k \theta_{2}$. Note that when $\lambda_{2}=$ $0, \lambda_{1}=\lambda, \theta_{1}=\theta,\left|W_{20}\right|=|W|$ and $\mu \gg k \theta$, (2) becomes identical with the BCS equation for $T_{c}$ of an elemental SC, and (1) leads to $|W|=2 k \theta /[\exp (1 / \lambda)-1]$ $(\Delta=k \theta / \sinh (1 / \lambda))$, where in the parentheses is noted the BCS equation for $\Delta$. Via a detailed comparative study of these equations for six elemental SCs [4], it has been shown that the equation for $|\mathrm{W}|$ provides a viable alternative to the equation for $\Delta$. We note that $s^{ \pm}$-wave feature is an inbuilt feature of (1) since it has been derived by assuming that the signature of $W_{20}$ changes on crossing the Fermi surface. Such an assumption leaves the BCS equation for $\Delta$ unchanged because it is quadratic in $\Delta$.

\subsection{Critical Current Density $j_{0}$ of an $S C$ at $T=0$ via CA in the 2PEM Scenario}

It has been shown that [6],

$$
\begin{gathered}
s\left(E_{F}\right) \equiv m^{*} / m_{e}=A_{1}\left(\gamma / v_{g}\right)^{2 / 3} E_{F}^{-1 / 3}, \\
n_{s}\left(E_{F}\right)=A_{2}\left(\gamma / v_{g}\right) E_{F}, \\
P_{0}\left(E_{F}\right)=A_{3}(\theta / y)\left(\gamma / v_{g}\right)^{1 / 3} E_{F}^{-2 / 3}, \text { and } \\
v_{0}\left(E_{F}\right)=A_{4}(\theta / y)\left(\gamma / v_{g}\right)^{-1 / 3} E_{F}^{-1 / 3} \text { hence, from the definition, } \\
j_{0}=\left(n_{s} / 2\right) e^{*} v_{0}\left(v_{0}=P_{0} / 2 m^{*}\right), \text { it follows that }[6][7]: \\
j_{0}=A_{5}(\theta / y)\left(\gamma / v_{g}\right)^{2 / 3} E_{F}^{2 / 3}\left(A_{5}=6.146 \times 10^{-4} \mathrm{C} \cdot \mathrm{eV}^{-4 / 3} \cdot \mathrm{K}^{1 / 3} \cdot \mathrm{s}^{-1}\right),
\end{gathered}
$$

where

$$
\begin{gathered}
A_{1} \simeq 3.305 \times 10^{-10} \mathrm{eV}^{-1 / 3} \cdot \mathrm{cm}^{2} \cdot \mathrm{K}^{4 / 3}, \\
A_{2} \simeq 2.729 \times 10^{7} \mathrm{eV}^{-2} \cdot \mathrm{K}^{2}, \\
A_{3} \simeq 1.584 \times 10^{-6} \mathrm{eV}^{4 / 3} \cdot \mathrm{cm} \cdot \mathrm{K}^{-1 / 3}, \text { and } \\
A_{4} \simeq 1.406 \times 10^{8} \mathrm{eV}^{2 / 3} \cdot \mathrm{sec}^{-1} \cdot \mathrm{K}^{-5 / 3}
\end{gathered}
$$

In these equations, $\theta$ is the Debye temperature of the SC and $\theta_{1}$ and $\theta_{2}$ the Debye temperatures of ion-species that cause pairing, $m^{*}\left(m_{e}\right)$ is the effective (free) electron mass, $\gamma$ the electronic specific heat constant and $v_{g}$ the gram-atomic volume of the SC; $\left(n_{s} / 2\right), e^{\star}$ (twice the electronic charge), $v_{0}$, and $P_{0}$ are, respectively, the number density, electronic charge, critical velocity and critical momentum of CPs (momentum at which $\Delta$ vanishes), and $y=\left(k \theta / P_{0}\right) \sqrt{2 m^{*} / E_{F}}$, a dimensionless construct to be obtained by solving $1 \cong \lambda_{1}\left[r_{1} y \ln \left\{r_{1} y /\left(r_{1} y-1\right)\right\}+\ln \left(r_{1} y-1\right)\right]+\left(\lambda_{1} \rightarrow \lambda_{2}, r_{1} \rightarrow r_{2}\right)$, where $r_{i}=\theta_{i} / \theta$.

This equation is derived via a BSE with the same kernel as employed for (1) and (2), except that now

$$
V_{i}=0 \text {, unless } E_{F}-k \theta_{i} \leq\left(\boldsymbol{P}_{0} / 2+\boldsymbol{p}\right)^{2} / 2 m^{*},\left(\boldsymbol{P}_{0} / 2-\boldsymbol{q}\right)^{2} / 2 m^{*} \leq E_{F}+k \theta_{i} .
$$

A more accurate (but rather elaborate) equation that additionally contains $E_{F}$ explicitly has been derived in [8]; the values of $y$ obtained via these equations differ significantly only when $\mu / k \theta \approx 0.3$ or less. 


\subsection{CA at Work, as Exemplified via Applications to a Cuprate and an Iron-Based SC}

1) $\mathrm{Tl}_{2} \mathrm{Ba}_{2} \mathrm{CaCu}_{2} \mathrm{O}_{8}$ (Tl-2212) [4] [6]: "Resolving" $\theta$ (Tl-2212) $=254 \mathrm{~K}$, we obtain $\theta_{\mathrm{Ca}}=254 \mathrm{~K}, \theta_{\mathrm{Tl}}=289 \mathrm{~K}, \theta_{\mathrm{Ba}}=296 \mathrm{~K}$. Assuming that pairing is due to the Ca and $\mathrm{Tl}$ ions and treating $\mu$ as an independent variable, we find via (1) and (2) that the empirical values $\left|W_{20}\right|=23.9 \mathrm{meV}$ and $T_{c}=110 \mathrm{~K}$ of the SC can be explained by a multitude of $\left\{\mu, \lambda_{\mathrm{Ca}}, \lambda_{\mathrm{T}\}}\right\}$-values. For each such set of values, we solve the equation for y given above and then calculate $j_{0}$ via (3), which leads to a multitude of values for the larger set $\left\{\mu, \lambda_{\mathrm{Ca}}, \lambda_{\mathrm{Tl}}, y, j_{0}\right\}$. Appeal to the empirical value, $j_{0}=6.5 \times 10^{7} \mathrm{~A} / \mathrm{cm}^{2}$, then fixes the set as: $\left\{\mu=49.8 \mathrm{meV}, \lambda_{\mathrm{Ca}}=0.4899, \lambda_{\mathrm{Tl}}=\right.$ 0.4543, $y=1.512\}$. As predictions, we have: $s=4.23, n_{s}=4.39 \times 10^{20} \mathrm{~cm}^{-3}, v_{0}=$ $9.35 \times 10^{5} \mathrm{~cm} / \mathrm{sec}$. Repetition of the above procedure for pairing via the $\mathrm{Ca}$ and $\mathrm{Ba}$ ions, or the $\mathrm{Ba}$ and $\mathrm{Tl}$ ions, fixes $\lambda_{\mathrm{Ba}}$. Each of the sets $\left\{\theta_{\mathrm{Ca}}, \lambda_{\mathrm{Ca}}\right\},\left\{\theta_{\mathrm{Ba}}, \lambda_{\mathrm{Ba}}\right\}$ and $\left\{\theta_{\mathrm{T} 1}, \lambda_{\mathrm{T} 1}\right\}$ then leads to a value of $\left|W_{10}\right|$ in the 1PEM scenario, and to $\left|W_{30}\right|$ in the 3PEM scenario when all these sets operative simultaneously.

2) $\mathrm{Ba}_{0.6} \mathrm{~K}_{0.4} \mathrm{Fe}_{2} \mathrm{As}_{2}$ (BaAs) [4] [5]: We obtain $\theta_{\mathrm{Ba}}=124.6 \mathrm{~K}, \theta_{\mathrm{Fe}}=399.4 \mathrm{~K}$ and $\theta_{\mathrm{As}}=$ $148.6 \mathrm{~K}$ from $\theta_{\mathrm{BaAs}}=274 \mathrm{~K}$. From among the multitude of empirical gap-values that characterize it, , e.g., $\approx 0,2.5,3.3,3.6,4,6,7,7.6,8.5,9,12 \mathrm{meV}$, we choose 6 and $12 \mathrm{meV}$ as our starting point and take its $T_{c}$ as $37 \mathrm{~K}$, which are also the values commonly employed in MBA. We now assume that while the smaller gap and the $T_{c}$ are due to the $\mathrm{Ba}$ and $\mathrm{Fe}$ ions, the larger gap pertains to the 3PEM scenario (involving also the As ions). This necessitates supplementing (1) and (2) by another equation, which follows from (1) by replacing $\left|W_{20}\right|$ by $\left|W_{30}\right|$ and adding $\left(\left|W_{30}\right| ; \lambda_{1} \rightarrow \lambda_{3} ; \theta_{1} \rightarrow \theta_{3}=\theta_{A s}\right)$ to its LHS; $\theta_{2}=\theta_{\mathrm{Fe}}$ on the RHS remains unchanged because it is greater than either of $\theta_{1}\left(=\theta_{\mathrm{Ba}}\right)$ and $\theta_{2}\left(=\theta_{\mathrm{As}}\right)$. By solving three simultaneous equations, viz., (1), (2), and (3), as against the normal practice of appealing only to the $T_{c}$ and $\Delta$-values of the SC, we are now led to a multitude of values for the set $\left\{\mu, \lambda_{\mathrm{Ba}}, \lambda_{\mathrm{Fe}}, \lambda_{\mathrm{As}}, y, j_{0}\right\}$. Appeal to $j_{0}=2.5 \times 10^{7} \mathrm{Acm}^{-2}$ then fixes this set as $\left\{\mu=14.2 \mathrm{meV}, \lambda_{\mathrm{Ba}}=0.1155, \lambda_{\mathrm{Fe}}=0.3838, \lambda_{\mathrm{As}}=0.2196, y=\right.$ $3.433\}$. Besides, we are thus led to a quantitative explanation of several empirical features of the SC, such as: $E_{P} / k T_{c}=4.45$, gap-values other than 6 and $12 \mathrm{meV}$, e.g., $\approx 0$ and $9 \mathrm{meV},\left(T_{c}\right)_{\max }$ (via $3 \mathrm{PEM}$ ) as exceeding $50 \mathrm{~K}$, and the "dome-like" structure of its $T_{c}$ vs. a tuneable variable; and, as predictions, to values of $s, n_{s}, V_{0}$, and coherence length $\xi$ as:

$$
s=0.420, n_{s}=3.1 \times 10^{20}\left(\mathrm{~cm}^{-3}\right), v_{0}=50 \times 10^{4} \mathrm{~cm} / \mathrm{sec}, \xi=7(\AA) .
$$

\section{Discussion and Conclusions}

1) CA satisfies the criteria noted for MBA in Section 2 because: (1) and (2) hold for arbitrary values of $E_{F}$ the ions responsible for pairing have been distinguished by distinct $\theta$ - and $\lambda$-values and the valence band overlapping the undulating Fermi surface has been characterized by locally spherical values-reminiscent of the locally inertial frames employed in the general theory of relativity [9]. We recall that even though none of the elemental SCs has a perfectly spherical Fermi 
surface [10], such an assumption works for them-barring a few for which $2 \Delta_{0} / k T_{c} \neq 3.52$. Finally, akin to MBA, CA too has been valuably employed for a wide variety of SCs, viz., several elemental SCs, $\mathrm{MgB}_{2}, \mathrm{YBCO}, \mathrm{Bi}-2212$ and Tl-2212 [4] [6] [7], $\mathrm{SrTiO}_{3}, \mathrm{La}_{2} \mathrm{CuO}_{4}, \mathrm{CeCoIn}_{5}$ [4], $\mathrm{Ba}_{0.6} \mathrm{~K}_{0.4} \mathrm{Fe}_{2} \mathrm{As}_{2}$ [4] [5], and $\mathrm{NbN}[8]$.

2) A salient feature of $\mathrm{CA}$ is that it invariably appeals to the ion-species that comprise an SC, whereas the number of bands invoked in MBA for the same SC differs from author to author [5]. Besides, by employing as input the values of any two gaps of an SC, CA goes on to shed light on several others, and puts its $\Delta s, T_{c}$ and $j_{0}$ under the same umbrella - which are features not shared by MBA.

3) While (3) identifies the parameters that can enhance $j_{0}$, and hence $T_{c}$ [11], of an SC, their optimization in practice is not straightforward because, while $\mathrm{y}$ depends on $E_{F}$ so do its constituents $m^{*}$ and $P_{0}$. Besides, any attempt to increase the value of $\left(\gamma / V_{g}\right)$, which is also implicitly a function of $E_{F}$ is bound to raise the problem of stability of the SC. Hence, in the quest for tangible clues to raise $T_{c} s$ of SCs, we need to have a comprehensive catalogue that includes, besides their $T_{c^{-}}$and $\Delta$-values, the values of $\theta, j_{0}, m^{\star}, v_{0}, n_{s}, \gamma$ and $v_{g}$

4) To conclude, with $s^{ \pm}$-wave as an intrinsic feature of it, we have shown that CA transforms the problem of raising $T_{c}$ into one of chemical engineering and that it is applicable to a wide variety of SCs, including the Fe-based SCs-without invoking a new state for them, as has been suggested via MBA [12]. Hence there is a need for its greater dissemination. Finally, both the approaches (without excluding others) need to be followed up since the cherished goal of room-temperature superconductivity may be reached by appealing to different sets of axioms-as in Euclidean geometry.

\section{Acknowledgements}

The author thanks D. C. Mattis, D. M. Eagles, A. Bianconi, R. Hott and A. Semenov for valuable correspondence, and L. K. Pande for a critical reading of the manuscript.

\section{References}

[1] Bianconi, A. (2005) Feshbach Shape Resonance in Multiband Superconductivity in Heterostructures. Journal of Superconductivity, 18, 625-636. https://doi.org/10.1007/s10948-005-0047-5

[2] Suhl, H., Matthias, B.T. and Walker, L.R. (1959) Bardeen-Cooper-Schrieffer Theory in the Case of Overlapping Bands. Physical Review Letters, 3, 552-554. https://doi.org/10.1103/PhysRevLett.3.552

[3] McMillan, W.L. (1968) Transition Temperature of Strong-Coupled Superconductors. Physical Review Journal Archive, 167, 331-344. https://doi.org/10.1103/PhysRev.167.331

[4] Malik, G.P. (2016) Superconductivity: A New Approach Based on the Bethe-Salpeter Equation in the Mean-Field Approximation. In: Directions in Condensed Matter Physics, Vol. 21, World Scientific, Singapore. 
https://doi.org/10.1142/9868

[5] Malik, G.P. (2017) A Detailed Study of the Role of Fermi Energy in Determining Properties of Superconducting NbN. Journal of Modern Physics, 8, 99-109. https://doi.org/10.4236/jmp.2017.81009

[6] Malik, G.P. (2016) On the Role of Fermi Energy in Determining Properties of Superconductors: A Detailed Comparative Study of Two Elemental Superconductors ( $\mathrm{Sn}$ and $\mathrm{Pb}$ ), a Non-Cuprate $\left(\mathrm{MgB}_{2}\right)$ and Three Cuprates (YBCO, Bi-2212 and Tl-2212). Journal of Ssuper-Conductivity and Novel Magnetism, 29, 2755-2764. https://doi.org/10.1007/s10948-016-3637-5

[7] Malik, G.P. (2018) Correction to: On the Role of Fermi Energy in Determining Properties of Superconductors: A Detailed Comparative Study of Two Elemental Superconductors $(\mathrm{Sn}$ and $\mathrm{Pb})$, a Non-Cuprate $\left(\mathrm{MgB}_{2}\right)$ and Three Cuprates (YBCO, Bi-2212 and Tl-2212). Journal of Superconductivity and Novel Magnetism, 31, 941-941. https://doi.org/10.1007/s10948-016-3637-5

[8] Malik, G.P. (2017) On the $s^{ \pm}$-Wave Superconductivity in the Iron-Based Superconductors: A Perspective Based on a Detailed Study of $\mathrm{Ba}_{0.6} \mathrm{~K}_{0.4} \mathrm{Fe}_{2} \mathrm{As}_{2}$ via the Generalized-Bardeen-Cooper-Schrieffer Equations Incorporating Fermi Energy. Open Journal of Composite Materials, 7, 130-145. https://doi.org/10.4236/ojcm.2017.73008

[9] Cracknell, A.P. and Kong, K.C. (1973) The Fermi Surface. Clarendon Press, Oxford.

[10] Weinberg, S. (1972) Gravitation and Cosmology: Principles and Applications of the General Theory of Relativity. John Wiley \& Sons, NY.

[11] Semenov, A., et al. (2009) Optical and Transport Properties of Ultrathin NbN and Nanostructures. Physical Review B, 80, 054510. https://doi.org/10.1103/PhysRevB.80.054510

[12] Bang, Y. and Stewart, G.R. (2017) Superconducting Properties of the $s^{ \pm}$-Wave State: Fe-Based Superconductors. Journal of Physics: Condensed Matter, 29, Article ID: 123003. https://doi.org/10.1088/1361-648X/aa564b 